\title{
Analysis of Patients' Perceptions Living with Implantable Cardioverter Defibrillators on Decision-Making, Satisfaction, Quality, and End of Life: Is there a Difference Between Primary and Secondary Prevention?
}

\author{
Swaroop Varghese ${ }^{1 *}$, Christoph Geller ${ }^{2}$ and Marc-Alexander Ohlow ${ }^{3}$ \\ ${ }^{1}$ Division of Cardiology, Ameosklinikum Halberstadt, Germany \\ ${ }^{2}$ Division of Cardiology, Zentralklinik, Bad Berka, Germany \\ ${ }^{3}$ Division of Cardiology, SRH Wald-Klinikum, Gera, Germany
}

Submission: January 28, 2021; Published: February 24, 2021

*Corresponding author: Swaroop Varghese, Division of Cardiology, Ameosklinikum Halberstadt, Germany

Abstract

Aims: The psychological impact of ICDs results in severe physical and psychosocial impairment. This study attempts to analyze patients' perceptions on decision making, satisfaction, security and end of life (EOL) as well as explore the similarities and differences between primary and secondary prophylaxis groups.

Methods and Results: ICD recipients were asked to complete a questionnaire during routine follow-up (FU) visits, their quality of life (QoL) was assessed with the Minnesota Living with Heart Failure Questionnaire (MLHFQ) and with the Hospital Anxiety and Depression Scale (HADS). Out of 423 patients (41\%CRT-ICD), 349(83\%) were recipients with primary prevention indication. The median age was 69 years. Seventy-one $(17 \%)$ patients reported a shock during a mean FU of $64 \pm 44$ months. While most patients $(90 \%)$ felt that their decision was right, younger patients ( $<69$ years) were doubtful. Though no major differences between the two groups were observed, those in secondary prevention group were more in favor of ICDs. With an overall moderate to poor quality of life and mild psychiatric symptoms (Mean QoL-score 29.4 \pm 20.3 , mean HADS-Score 8.7 \pm 7.3 ), there were no significant differences between both groups. Although 361 (87.4\%) patients felt secure and safe, those with shocks were significantly more insecure. Only a minority of patients (31\%), mostly younger, considered ICD-deactivation during EOL with no notable difference between the groups.

Conclusion: Most patients felt safer following ICD implantation judging their decision for ICD as right. Only a minority considered ICDdeactivation at end of life situations. There were no significant differences between primary and secondary prevention groups.

Keywords: Implantable cardioverter-defibrillator; Patient-reported outcomes; Satisfaction with treatment; Patient perception; End of Life situations

\section{Introduction}

The therapeutic benefits of implantable cardioverter defibrillators (ICD) in the management of sudden cardiac deaths have been proven beyond doubt. With significant scientific evidence supporting the ICDs, current international and national guidelines strongly endorse the device in high-risk patients with either ischemic or non-ischemic heart failure [1]. This has resulted in a significant increase in implants, with 252 implants in Europe and 675 implants in the USA per million of the population per year $[2,3]$.

Despite the wide acceptance of ICDs [4], there have been growing concerns regarding the adverse patient-reported outcomes after ICD implantation [5]. The necessity of regular follow-ups, concerns of device or lead malfunction, receiving shocks (both appropriate and inappropriate), manufacturer recalls as well as lifestyle restrictions severely impact the quality of life (QoL), causing significant psychological distress to patients [6]. Psychiatric issues, predominantly depression and anxiety, have been reported affecting up to $87 \%$ of patients 
post-implantation invariably impairing physical, social, and psychological functioning $[3,7,8]$.

Various studies have assessed the multidimensional aspects of psychosocial impact and their sequelae in patients post ICD implantation $[3,7,8,9]$, emphasizing the role of psychological interventions in the long-term management of patients. While the ICDs may be an absolute necessity in secondary prevention, the impact of primary prophylaxis implantation on adverse patientcentered outcomes such as QoL and distress has been subject to debate [10].

The wide array of psychological challenges following ICD implantation mandate critical discussions on patients' perceptions in terms of decision making, sense of security, satisfaction as well as end of life (EoL) situations. Furthermore, analyzing the similarities and differences of these reported outcomes between both primary and secondary preventive indications may be instrumental in informing caregivers, influencing decisions, and providing tailored, patient-oriented care, thereby maximizing health outcomes across all dimensions.

Therefore, our research intends to evaluate the perceptions of ICD patients in primary and secondary prevention, explore their similarities and differences in their reported outcomes and enhance our understanding of patient satisfaction, sense of security, and EoL situations.

\section{Methods}

In our cross-sectional designed single-center study, consecutive ICD recipients, implanted recently or in the past, between January 2007 and December 2010 at the Zentralklinik Bad Berka, Germany, were included. The indications for ICD implantation were based on the European guidelines [1]. These guidelines recommend an ICD implantation in ischemic or nonischemic heart failure with a reduced left ventricular ejection fraction (LVEF) $\leq 35 \%$ (primary prevention) or a documented ventricular fibrillation or ventricular tachycardia with hemodynamic instability (secondary prevention).

The recipients are usually scheduled every 3 - 6 months for regular follow-ups at the ICD outpatient clinic in Zentralklinik, Bad Berka. Additional visits are warranted following ICD discharges. During the regular follow-ups, the outpatients were informed by the physician about the study, which was formally a survey intended to enquire about their subjective responses across several variables considered relevant to the research.

While the in-patients were informed in their rooms, the acutely ill patients were excluded from the study. Upon giving oral informed consent, the patients were consecutively enrolled. Demographic, clinical, and follow-up data were collected from the previous hospital records.

\section{Ethics Declaration}

This study has been approved by the institutional ethics committee and has therefore been performed by the ethical standards laid down in the 1964 Declaration of Helsinki and its later amendments.

\section{Materials}

A carefully drafted self-administered questionnaire in the native language was conceptualized for the study designed to analyze the subjective responses of patients who received an ICD. Care was taken to formulate simple questions to enhance patient comprehension, and they were encouraged to return the forms only after fully completing them. Additionally, three experienced nurses offered to assist the patients in clarifying their queries and also ensuring an almost $100 \%$ completion of the forms.

The questionnaire comprised of 3 parts:

1. The first part consisted of a set of mostly pre-coded questions aimed at defining demographic characteristics, such as age, sex, educational levels, number of people per household.

2. Clinical variables included ICD indication (primary vs. secondary prevention), current functional status in heart failure based on the New York Heart Association (NYHA) for heart failure and the Canadian Cardiovascular Society (CCS) for angina pectoris as well as the presence (or absence) of psychiatric illnesses such as depression, anxiety, and schizophrenia.

3. Device related variables such as consent before the procedure, the type of device implanted, complications, number of redo-procedures, as well as the desire of a psychological intervention after device implantation, number of shocks were derived both from subjects as well as medical records. Lastly, the patients' current perspectives on their decision for implantation, their perceptions on safety with the device, and their views on deactivating the ICD in end-of-life situations were also enquired.

4. The clinical data related to heart failure and associated comorbidities, as well as current drug history, were collected from previous hospital records. The relevant variables have been outlined under the corresponding columns in the table below.

5. The second part of the questionnaire - Minnesota Living with Heart Failure Questionnaire (MLHFQ), translated version: MLHFQ is a self-assessment questionnaire of 21 items representing - a) physical, b) emotional, and c) socioeconomic factors in heart failure (HF) that negatively impact the quality of life. The variables are ranked from 0 (none) to 5 (very much) on a six-point Likert scale, which subsequently indicates the severity of $\mathrm{HF}$, which restricted the patient from living as he/she would have otherwise wanted to during the past four weeks. 
The questionnaire is tallied by summating the 21 responses in total [11]. The overall score ranges from 0 to 105 , indicating the best to worst HF-related quality of life. Out of the 21 questions, the MLHFQ also provides individual scores for two components, namely physical -8 items with a range between $0-40$ and emotional - five items with a range of 0 - 25. The remaining eight items are merely applied for the calculation of the total score [11]. The grading of the overall scores are good $(<24$ points), moderate (25- 45 points), and poor QoL ( $>45$ points). These cut-off values strongly correlate with survival, self-perceived health status, New York Heart Association (NYHA) classification, and 6 min walk test [12].

Across the various HF-related quality of life questionnaires, the MLHFQ is amongst the most popularly known and widely employed instruments. It has been translated and culturally adapted into several languages and has exhibited excellent psychometric properties in several studies [13], it is also highly sensitive to treatment-associated differences QOL [14]. The instrument can be used as a critical outcome measure in studies and evaluations of outpatients with symptomatic (NYHA II-IV) heart failure with a reduced or preserved ejection fraction. It can determine the effectiveness of a treatment in improving the quality of life by reducing the adverse impact of heart failure.

\section{The last part of the questionnaire included the Hospital Anxiety and Depression Scale (HADS)}

The HADS is a 14-item self-administered questionnaire designed to measure the symptoms of anxiety and depression in medical patients. The HADS comprises seven questions for anxiety and seven questions for depression, and these are scored separately. It takes 2-5 minutes to complete, and the items are ranked on a 4-point severity scale, delivering information on two different levels - Anxiety (HADS-A) and Depression (HADS-D) [15]. For both scales, scores of less than 7 indicate non-cases. Scores between 8 - 10 are regarded as mild, 11 - 14 moderate, and 15 - 21 severe. Values of more than 10 are usually referred to as cases [16]. The HADS is simple, easy to use, and allows for the assessment of both anxiety and depression, which usually tends to coexist $[17,18]$.

Cut-off scores are available for quantification, for example, a score of 8 or more for anxiety has a specificity of 0.78 and a sensitivity of 0.9 , and for depression a specificity of 0.79 and a sensitivity of 0.83 [19].

Patients also do not have any difficulty in understanding the reason for the request to answer the questionnaire. The validity of the HADS has been confirmed in many studies [20].

\section{Statistical Analysis}

The extracted data were entered into an Excel Spreadsheet. Continuous variables are reported as mean value \pm standard deviation or median or interquartile ranges (25th-75th percentiles) as appropriate. Categorical variables are presented as absolute (n) and relative (\%) frequencies. The normal distribution of variables was assessed using the D'Agostino-Pearson omnibus normality test. The T-test and Fisher's exact test were used as appropriate. All tests were two-tailed, and a probability value of $\mathrm{p}<0.05$ was considered statistically significant.

Statistical analysis was performed using the GraphPad Prism version 6.02 for Windows (Graph-Pad Software, La Jolla, CA, USA).

\section{Results}

\section{Patient population}

Out of 434 patients requested, 423 patients agreed to participate in the study ( $98 \%$ response rate). While the median age of our cohort was 69 years, there was an overall male predominance (342 patients, 81\%). Regarding educational levels, $38 \%$ of patients had completed primary school, $40 \%$ were high school graduates, and the rest $22 \%$ had higher levels of education. Most of the patients (83\%) reported being living with a partner.

\section{Clinical and device-related variables}

Out of 423 patients, 349 patients (83\%) were recipients with primary prevention (PP) indication. The most common underlying etiology was ischemic heart failure (80\%) with mean ejection fraction (EF) of $41 \pm 12 \%(39 \pm 12 \%$ PP vs. $49 \pm 12 \%$ in secondary prevention (SP), $\mathrm{p}<0.0001$ ) and the mean severity of heart failure symptoms according to New York Heart Association (NYHA) was estimated at $1.8 \pm 0.6$. Around $41 \%$ of the cohort had cardiac resynchronization therapy (CRT-ICD); the patients were mostly males (p-value 0.017 ), younger ( $<61$ years, $<0.0001$ ), and often in primary prevention groups ( $\mathrm{p}$-value $<0.0001$ ). The patients followed a guidelines-recommended heart failure therapy, namely beta-blockers (95\%) and angiotensin-converting enzyme inhibitors (ACEI)/angiotensin receptor blockers (ARB) (92\%) with comparatively lower rates $(50 \%)$ on aldosterone receptor antagonists. Those who experienced shocks were often on Amiodarone (p-value 0.0007).

Most patients (95\%) reported that they were adequately informed prior to implantation about the device, procedure, and possible complications. The mean interval from the time of implantation to the current follow up was $64 \pm 44$ months during which $37 \%$ reported late complications such as lead dysfunction, pocket associated problems, device/lead infection as well as other complications. Out of $17 \%$ of patients who experienced a shock, $8 \%$ reported multiple shocks with a slight preponderance of adequate shocks in the SP-group (p-value 0.02); Those with multiple shocks were usually younger patients ( $p$-value 0.0002 )

\section{Decision making, sense of security, and Patient satisfaction}

The majority of patients (90\%) regarded their decision for ICD as right, while the remaining (10\%) expressed their doubts. 
Though there were no statistically significant findings between the two groups, patients in the SP-group were more in favor of ICD implantation (93\% vs. $88 \%$ in primary prevention, p-value 0.85 ). Conversely, patients in PP were more doubtful of their decision when compared to those in SP (10\% vs. $7 \%$, p-value 0.77$)$.

Although the majority of the cohort (87.4\%) expressed overall satisfaction with a sense of security and safety, the impact of shocks significantly affected this perception ( $p$-value 0.0091). While 34 patients $(8.2 \%)$ reported not feeling secure with ICDs, this perception was pronounced in the SP group who received shocks. However, this lacked any statistical relevance. Out of the rest (4.4\%), younger patients ( $<61$ years, p-value 0.0036 ) significantly had mixed feelings about security; it was interesting, however, to note that, despite lacking statistical power, slightly more patients in the PP were having mixed feelings than those in SP (5\% vs. $3 \%$, p-value 0.6$)$.

\section{Information and attitude in the case of end-stage disease}

Only a minority of recipients (31\%) considered ICDdeactivation during near EOL situations with no significant difference noticed between PP and SP groups. Younger recipients more significantly considered the deactivation.

\section{Quality of Life (QoL) Assessment based on MLHFQ- Scores}

The cohort reported a moderate (to poor) quality of life with a mean score of $28.41 \pm 20.61$. This was noted particularly in younger patients ( $<61$ years, p-value 0.04 ) and in those who experienced shocks ( $\mathrm{p}$-value 0.01). Males reported to have a higher QoL, but only in the absence of shocks (p-value 0.003). Females had comparatively poor QoL (p-Value 0.002); those with poor QoL were significantly affected by shock-related events (p-value 0.0015). There were no relevant differences in QoL between primary and secondary prevention groups.

\section{Hospital Anxiety and Depression Scale (HADS)-Scores}

Although pre-existing psychiatric diseases such as depression and anxiety were prevalent in $9 \%$ of the cohort, they were more common in secondary prevention.

While our sample had a mean HADS-Score of 7.72 \pm 7.40 indicating mild symptoms, higher HADS-and anxiety-scores were reported in younger patients ( $p$-value 0.0018 ) as well as those who experienced shocks ( $\mathrm{p}$-value 0.03). Scores indicating depression were higher in younger patients. No relevant differences in HADS-scores were observed between primary and secondary prophylaxis groups.

\section{Discussion}

The main findings of our study may be summarized as follows:

a) The majority of the patients (90\%) regard their decision for ICDs as right while younger patients were more doubtful. Though statistically insignificant, those with primary prophylaxis were slightly more doubtful.

b) Moderate to poor QoL was observed in younger patients, females, and in those who had previous shocks. There were no relevant differences in QoL between PP and SP groups.

c) Patients who experienced shocks, as well as younger patients, were often anxious, younger patients additionally reported higher depression scores. The HADS-scores were comparable between both PP and SP-groups.

d) Although there is a high overall patient satisfaction (87.4\%) with a sense of security after ICD-implantation among both the groups, it may be ironically distorted in the event of shock-related incidents. Among those who felt insecure (8.2\%), the perception was more pronounced in patients from the SP group who experienced shocks. Fewer patients (4.4\%) had mixed feelings about ICDs; they were reported more in the PP group. However, both these findings lacked statistical relevance. Most patients (95\%) said that they were adequately informed before implantation about the device, procedure, and possible complications.

e) Only a minority of recipients (31\%) considered ICDdeactivation during near EOL situations. While the response was similar in both PP and SP-groups, the deactivation was significantly considered by younger patients.

\section{Decision-making in patients with ICD implantation}

The majority of our patients (90\%) regarded their decision for ICDs as right. However, this may be explained due to the majority being older patients. Older patients are aware of their morbidity and mortality and, therefore, more willing to accept the burdens of ICD therapy in return for the possibility of life extension [21]. They are also more likely to have lower health literacy, cognitive impairment, and sensory deficits that would make effective communication with their doctors challenging, therefore tempting them to rather follow the doctor's recommendation without further question. In contrast, doctors, inspired by pathbreaking trials, evidence, and guidelines, emphasize more on the benefits while minimizing potential harm $[22,23]$.

The remaining $10 \%$ either regretted or had doubts about their decision for ICD-implantation. While these patients were younger, these findings may also be attributed to recall bias, where the patients express disappointment with experiences occurring after the implantation. Since many of our patients had their ICDs for 4 - 5 years, their regret might have been influenced by living with the device and experiencing the trade-offs [22].

Our results also showed that, though statistically insignificant, those with doubts or who refused were slightly more in the primary prophylaxis group. Since the decision to implant ICDs are preference-sensitive considering the tradeoffs involved, this may 
be relevant in primary prophylaxis patients in whom the benefits of ICD are not clear. Many authors opine the necessity of elaborate discussions with patients explaining the indication of the ICDs, its benefits, and risks, potential consequences for the quality of life as well as alternative therapy options to ensure the decision-making process is consistent with patient preferences [22-24].

The European Society of Cardiology guidelines recommends the discussion of quality-of-life issues before ICD implantation and during disease progression in all patients (Class I C recommendation) [25]. Although the American College of Cardiology (ACC) and American Heart Association (AHA) also recommend shared decision making surrounding the use of technologies such as ICDs in patients with heart failure, there are substantial challenges in implementing these guidelines [26]. A group study in the recent past found that $80 \%$ of patients with ICDs did not remember having a discussion with their doctors about the associated risks and possible long-term complications after ICD implantation. There seems to be a consensus that the patients understood the ICD risks and benefits after implantation than before it [9].

In a cross-sectional study, Caverly et al. [24] showed that more than half of the physicians rated patient preferences lower than expected mortality benefits. The precedence of paternalistic physician behavior over patient preferences in the decisionmaking process would thereby conclude that the more convinced a physician is of the benefits of an intervention, the less critical patient preferences become in decision making [24]. Overall, our findings were consistent with those in previous studies [22,23,24]

\section{Quality of Life (QoL) in ICD patients}

Quality of Life is a broad concept that may fall short of a universal definition; however, many would concur that it is a generic term for a multi-dimensional health outcome in which biological, psychological and social functioning are interdependent [27].

Based on the MLHFQ-Scores, our findings showed moderate to poor QoL in younger patients ( $<61$ years), in females as well as in those who had previous (multiple) shocks.

Sears et al. reported that ICD patients $<50$ years of age defined in literature as young - have increased psychological distress, perhaps due to difficulties making lifestyle adjustments and due to additional stressors, such as fear of job security and loss of role functioning $[27,28]$.

Most studies also suggest that shocks negatively impact QOL [6]. In the AVID trial, the occurrence of even one shock was associated with a reduction in mental well-being and physical function, even after controlling for multiple clinical factors such as heart failure, index arrhythmia, and ejection fraction. Further, there was a more significant reduction in QOL as the number of shocks increased [29]. In the Canadian Implantable Defibrillator (CIDs) trial, although those patients who received either no shocks or one to four shocks had significant improvement in QOL over time, those with five or more shocks did not improve [30].

Poorer QoL was observed among females in our cohort, a pattern similar to the findings reported in a US national survey examining global QoL and psychosocial issues in ICD patients [31]. Interestingly, the number of single-person households were also higher among females in our sample. Low social support is also a predictor of poor QOL for patients with ICDs, in particular, the absence of a spouse [32,33].

There were no relevant differences in QoL between PP and SP groups in our study, consistent with the findings of a previous analysis of five different studies by Pedersen et al. [34].

\section{Anxiety and Depression}

Although depression and anxiety were present in $9 \%$ of our cohort (more common in SP-groups) at the time of our study, higher HADS-scores were found in younger patients as well those who had experienced shocks. While anxiety was more pronounced after shocks, younger patients additionally reported feeling depressed. Several authors have reported similar findings $[32,35,36]$. A systematic review of 45 studies that assessed $>$ 5000 patients reported between $11 \%$ to $28 \%$ of patients with depressive disorder and $11 \%$ to $26 \%$ had an anxiety disorder [36]. Posttraumatic stress disorder is also increased in patients with ICDs, with a recent single-center study of 308 ICD recipients describing a rate of $35 \%$ for significant anxiety or posttraumatic stress disorder after implantation [37].

In contrast to a large Swedish cross-sectional study [38] we did not find any relevant difference in anxiety and depression between male and female ICD recipients. This finding may be partly attributed to the fact that there were fewer female patients in our cohort, a limitation in several studies observed by Dunbar et al. [39].

Unlike the findings of a multicenter survey [40], our study showed interestingly similar HADS-scores between both primary and secondary prevention groups.

As with QoL, it is likely that many factors, such as baseline psychological traits and social support, interact with shocks to impact whether patients develop anxiety, depression, or posttraumatic stress disorder $[35,41]$.

\section{Information Satisfaction and feeling of Safety after ICD- implantation}

Despite the high psychosocial burden, acceptance of ICD is usually high, with only a few expressing negativities about the device $[41,42]$. The majority of the patients do well with their device, and positive life experiences after ICD implantation and high level of satisfaction with treatment have been reported $[39,43]$. With $95 \%$ of our patients expressing appreciation with the information provided prior to ICD implantation and majority indicating a sense of safety after implantation, our findings are 
consistent with previous similar studies [39,43,44]. However, it was interesting to note that the perceived sense of safety was distorted after shocks, and younger patients were somewhat unsure after implantation. This change in perception may be attributed to the anxiety and other (as mentioned previously) components which play a significant role in ICD patients. There was no relevant difference between primary and secondary prophylaxis groups.

\section{End of life situations}

Only a minority of recipients (31\%) considered ICDdeactivation during near End of Life situations. While the response was similar in both PP and SP-groups, the deactivation was significantly considered by younger patients. Our results were consistent with some of the other studies [45] while other studies reported a higher percentage of patients favoring ICD deactivation at the end of life $[46,47]$.

The European Society of Cardiology (ESC), Canadian Cardiovascular Society (CCS), American College of Cardiology (ACC), American Heart Association (AHA) and the Heart Rhythm Societies in Europe and America endorse a proactive approach to ICD deactivation [48-50].

Deactivation of the ICD is legal and morally acceptable when it is consistent with patient goals [51]. Twenty percent of ICD patients receive shocks in the last weeks, days, or hours of their lives, decreasing their QOL, and that of their families as well [52]. Turning off the defibrillator will avoid unnecessary and painful shocks at the end of life. Moreover, the option to turn off the defibrillator in patients with severe advanced heart failure presents the possibility that their mode of death may change from progressive heart failure to sudden death, which may be a preferable and less traumatic death for patients [53].

The ICD deactivation remains a difficult subject for both doctors and patients to discuss, especially when there are various competing comorbidities and factors contributing to their terminal illnesses. The ESC recommendations state that the ICD deactivation should be considered when clinical conditions deteriorate [54]. Greater awareness among physicians and health care personnel is therefore required, also ensuring the incorporation of patients' values and preferences in the final decision-making process [55].

\section{Limitations}

The findings of our study should be interpreted with some caution, as it is based on a single-center analysis. However, the response rate of $98 \%$ when compared to some of the recent studies with response rates of $81 \%$ and $51 \%[56,57]$ may somewhat add weight to our conclusions. Just like prior related studies, our cohort too had a male predominance, and the majority were in the primary prevention group. The impact of remote monitoring (RM) on the quality of life has not been primarily assessed in our study. Though RM has been employed in the advanced care of our patients. This has essentially not replaced our follow-up visits to the CIED clinics and hence not considered in our study. Also, the additional benefit of Angiotensin Receptor-Neprilysin inhibitors could not be evaluated in the overall impact on QoL in our patients. We did not have any patients with subcutaneous cardioverterdefibrillators. Lastly, due to the cross-sectional design of our study, only assessments following ICD-implantation were evaluated. We did not have the assessments before implantation; changes in the patient-reported outcome trajectories could not be assessed, which could lead to interpretation bias.

\section{Conclusion}

From a physicians' perspective, it may be heartening to see a high overall patient satisfaction after ICD implantation with a majority of them regarding their decision as right. However, this should not camouflage one from understanding and addressing the individual concerns of patients, especially those who are younger, who experienced shocks, perhaps even those with primary indication.

With growing psychosocial concerns surrounding ICD therapy, our results should encourage physicians to identify these "at-risk" patient groups. Physicians have to stratify those who may or may not benefit from ICDs as well as provide patients' timely psychological care and support, which may help improve outcomes, particularly Quality of Life.

Though fewer patients considered ICD deactivation during near End of Life situations, this topic warrants a more proactive approach from physicians explaining the course of ICD therapy in such events to provide appropriate terminal care suited to patients' desires.

\section{Acknowledgments}

Apart from thanking all the patients for participating in our study, we would also like to acknowledge the valuable work of the CIED nursing team: Christine Feser and Anne Hasch.

\section{Funding}

The authors declare that there are no relationships relevant to the contents of this paper to disclose. There was no relationship with the industry.

\section{Conflicts of interest: None declared.}

\section{References}

1. Stella BM, Alessandro, Z (2015) ICD Implantation Practice Within Europe: How to Explain the Differences Beyond Economy. J Atr Fibrillation 8(3): 1262.

2. Camm J, Nisam S (2011) Implantable cardioverter-defibrillator utilization. Europace 13(3): 448.

3. Pedersen S, Knudsen C, Dilling K, Sandgaard NCF, Johansen JB (2017) Living with an implantable cardioverter-defibrillator: patients preferences and needs for information provision and care options. Europace 19: $983-990$. 
4. Lüderitz B, Jung W, Deister A, Manz M (1994) Patient acceptance of implantable cardioverter-defibrillator devices: changing attitudes. Am Heart J 127(4 Pt 2): 1179-1184.

5. Yuhas J, Mattocks K, Gravelin L, Remetz M, Foley J, et al. (2012) Patients' Attitudes and Perceptions of Implantable Cardioverter-defibrillators. Pacing and Clinical Electrophysiology 35(10): 1179-1187.

6. Lampert R (2013) Managing with Pacemakers and Implantable Cardioverter Defibrillators. Circulation 128(14): 1576-1585.

7. Sola CL, Bostwick JM (2005) Implantable Cardioverter-Defibrillators, Induced Anxiety and Quality of Life. Mayo Clin Proc 80(2): 232-237.

8. Hoogwegt MT, Kupper N, Theuns DA, Zijlstra WP, Jordaens L, et al. (2012) Undertreatment of anxiety and depression in patients with an implantable cardioverter-defibrillator: impact on health status. Health Psychol 31(6): 745-753.

9. Hauptmann PJ, Chibnall JT, Guild C, Armbrecht ES (2013) Patient perceptions, physician communication and the implantable cardioverter-defibrillator. JAMA Intern Med 173(7): 571-577.

10. Pedersen SS, Sears SF, Burg MM, Van Den Broek KC (2009) Does ICD indication affect quality of life and levels of distress? Pacing Clin Electrophysiol 32(2): 153-156.

11. Palakunnel J, Nourbakhsh M, Nia SFM, Stan AC, Sardar H, et al. (2019) Translation and validation of the Minnesota Living with Heart Failure Questionnaire for the Spanish speaking population residing in the United States. Journal of Heart and Cardiology 2019.

12. Behlouli H, Feldman DE, Ducharme A, Frenette M, Gianetti N, et al. (2009) Identifying relative cut-off scores with neural networks for interpretation of the Minnesota Living with Heart Failure questionnaire. Conf Proc IEEE Eng Med Biol Soc 6242-6246.

13. Bilbao A, Escobar A, Garcia-Perez L, Navarro G, Quiros R (2016) The Minnesota living with heart failure questionnaire: comparison of different factor structures. Health Qual Life Outcomes 14: 23.

14. Rector TS, et al. (1995) Use of Living with heart Failure questionnaire to ascertain patientsÄ perspective on improvement in quality of life versus risk of dug induced death. J Card Fail 1: 201-206.

15. Edelstein BA, Ciliberti CM (2010) Assessment of Depression and Bereavement in Older Adults. s.l. : Handbook of Assessment in Clinical Gerontology.

16. Varghese S, Geller JC, Ohlow MA (2019) Phantom shocks in implantable cardioverter-defibrillator recipients: impact of education level, anxiety, and depression. Herzschrittmachertherapie und Elektrophysiologie 30(3): 306-312.

17. McManus S, Meltzer H, Brugha T, Bebbington P, Jenkins R (2014) Adult Psychiatric Morbidity in England: Results of Household Survey.

18. Stern A (2014) The Hospital Anxiety and Depression Scale. Occup Med 64(5): 393-394

19. Bjelland I, Dahl AA, Haug TT, Neckelmann D (2002) The validity of the Hospital Anxiety and Depression Scale. An updated literature review. J Psychosom Res 52(2): 69-77.

20. Snaith RP (2003) The Hospital Anxiety and Depression Scale. Health Qual Life Outcomes 1: 29.

21. Finucane TE (1999) How gravely ill becomes dying: a key to end-of-life care. JAMA 282(17): 1670-1672.

22. Green AR, Jenkins A, Masoudi FA, Magid DJ, Kutner JS, et al. (2016) Decision-Making Experiences of Patients with Implantable Cardioverter-Defibrillators. Pacing Clin Electrophysiol 39(10): 10611069.

23. Matlock DD, Nowels CT, Masoudi FA, Sauer WH, Bekelman DB, et al.
(2011) Patient and cardiologist perceptions on decision making for implantable cardioverter-defibrillators: a qualitative study. Pacing Clin electrophysiol 34(12): 1634-1644.

24. Caverly TJ, Al-Khatib SM, Matlock DD (2012) Patient Preference in the Decision to place Implantable Cardioverter-Defibrillators. Arch Intern Med 172(14): 1104-1105.

25. Priori SG, Blomström-Lundqvist C, Mazzanti A, Blom N, Borggrefe M, et al. (2015) 2015 ESC guidelines for the management of patients with ventricular arrhythmias and the prevention of sudden cardiac death. Europace 17(11): 1601-1687.

26. Yancy CW, Jessup M, Bozkurt B, Butler J, Casey DE Jr, et al. (2013) ACCF/AHA Guideline for the management of heart failure: a report of the American College of Cardiology/AHA Task Force on Practice Guidelines. J Am Coll Cardiol 62(16): e147-239.

27. Sears Jr, Samuel F, Conti JB (2002) Quality of Life and Psychological Functioning of ICD patients. Heart 87(5): 488-493.

28. Sears SF Jr, Burns JL, Handberg E, Sotile WM, Conti JB (2003) Young at heart: understanding the unique psychosocial adjustment of young implantable cardioverter defibrillator recipients. Pacing Clin Electrophysiol 24(7): 1113-1117.

29. Schron EB, Exner DV, Yao Q Jenkins LS, Steinberg JS, et al. (2002) Quality of life in the Antiarrhythmics versus Implantable Defibrillators trial. Circulation 105(5): 589-594.

30. Irvine J, Dorian P, Baker B, O’Brien BJ, Roberts R, et al. (2002) Quality of life in the Canadian Implantable Defibrillator Study (CIDS). Am Heart J 144(2): 282-289.

31. Sears SF (1999) The U.S. national survey of ICD recipients: examining the global and specific aspects of quality of life. European Heart Journal 20: 232 .

32. Sears SF, Leiws TS, Kuhl EA, Conti JB (2005) Predictors of quality of life in patients with implantable cardioverter-defibrillators. Psychosomatics 46(5): 451-457.

33. Luyster FS, Hughes JW, Waechter D, Josephson R (2006) Resource loss predicts depression and anxiety among patients treated with an implantable cardioverter-defibrillator. Psychosom Med 68(5): 794800 .

34. Pedersen SS, Sears SF, Burg MM, Van den Broek KC (2009) Does ICD indication affect quality of life and levels of distress? Pacing Clin Electrophysiol 32(2): 153-156.

35. Dunbar SB, Dougherty CM, Sears SF, Carroll DL, Goldstein NE, et al. (2012) Educational and psychological interventions to improve outcomes for recipients of implantable cardioverter defibrillators and their families. Circulation 126(17): 2146-2172.

36. Magyar-Russell, G, Thrombs BD, Cai JX, Baveja T, Kuhl EA, et al. (2011) The prevalence of anxiety and depression in adults with implantable cardioverter-defibrillators: a systematic review. J Psychosom Res 71(4): 223-231.

37. Kapa S, Rotondi-Trevisan D, Mariano Z, Aves T, Irvine J, et al. (2010) Psychopathology in patients with ICDs over time: results of a prospective study. Pacing Clin Electrophysiology 33(2): 198-208.

38. Thylen L, Dekker RL, Jaarsma T, Strömberg A, Moser DK (2014) Characteristics associated with anxiety, depressive symptoms and quality of life in a large cohort of implantable cardioverter-defibrillator recipients. J Psychosom Res 77(2): 122-127.

39. Dunbar S, Dougherty CM, Sears SF, Carroll DL, Goldstein NE, et al (2012) Educational and Psychological Interventions to Improve Outcomes for Recipients of Implantable cardioverter Defibrillators and Their Families. Circulation 126(17): 2146-2172. 
40. Rahmawati A, Chishaki A, Ohkusa T, Sawatari H, Tsuchihashi-Makaya $M$, et al. (2016) Influence of primary and secondary prevention indications on anxiety about implantable cardioverter-defibrillator. J Arrhythm 32(2): 102-107.

41. Pedersen SS, Van den Broek KC, Van den Berg M, Theuns D (2010) Shock as a Determinant of Poor Patient-Centered Outcomes in Implantable Cardioverter Defibrillator Patients: Is there More to It than meets the Eye. Pacing Clin Electrophysiol 33(12): 1430-1436.

42. Dunbar SB (2005) Psychosocial Issues of Patients with Implantable Cardioverter Defibrillators. Am J Crit Care 14(4): 294-303.

43. Lüderitz B, Jung W, Deister A, Manz M (1997) Patient acceptance of implantable cardioverter-defibrillator devices: changing attitudes. American Heart Journal 127(4 Pt 2): 1179-1184.

44. Pedersen SS, Knudsen C, Dilling K, Sandgaard NCF, Johansen JB (2017) Living with an implantable cardioverter-defibrillator: patients' preferences and needs for information provision and care options Europace 19(6): 983-990.

45. Herman D, Stros P, Curila K, Kebza V, Osmancik P (2013) Deactivation of implantable cardioverter-defibrillators: results of patient surveys. Europace 15(7): 963-969.

46. Stewart GC, Weintraub JR, Pratibhu PP, Semigrn MJ, Camuso JM, et al. (2010) Patient expectations from implantable defibrillators to prevent death in heart failure. J Card Fail 16(2): 106-113.

47. Pedersen SS, Chaitsing R, Szii-Torok T, Jordaens L, Theuns DA (2013) Patients' perspective on deactivation of the implantable cardioverterdefibrillator near the end of life. Am J Cardiol 111(10): 1443-1447.

48. Dodson JA, Fried TR, Lampert R (2013) Patient Preferences for Deactivation of Implantable Cardioverter Defibrillators. JAMA Intern Med 173(5): 377-379.

49. Lampert R, Hayes DL, Annas GJ, Farkey MA, Goldstein NE, et al. (2010) HRS Expert Consensus Statement on the Management of Cardiovascular Implantable Electronic Devices (CIEDs) in patients nearing end of life or requesting withdrawal of therapy. Heart Rhythm 7(7): 1008-1026.
50. Arnold JM, Liu P, Demers C, Dorian P, Gianetti N, et al. (2006) Canadian Cardiovascular Society consensus conference recommendations on heart failure 2006: diagnosis and management. Can J Cardiol 22(1): 23-45.

51. Jessup M, Abraham WT, Casey D, Feldman AM, Francis GS, et al. (2009) 2009 focused update: ACCF/AHA guidelines for the diagnosis and management of heart failure in adults: a report of the American College of Cardiology Foundation/AHA Association task force on practice guidelines. Circulation 119(14): 1997-2016.

52. Mitar M, Alba A, MacIver J, Ross H (2012) Lost in Translation: examining patient and physician perceptions of implantable cardioverterdefibrillator deactivation discussions. Circ Heart Failure 5(5): 660-666.

53. Goldstein NE, Lampert R, Bradley E, Lynn J, Krumholz HM (2004) Management of implantable cardioverter-defibrillators in end-of-life care. Ann Intern Med 141(11): 835-838.

54. Maclver J, Tibbles A, Billia F, Ross H (2016) Patient perceptions of implantable cardioverter-defibrillator deactivation discussions: A qualitative study. SAGE Open Medicine 4: 1-8.

55. Priori SG, Blomström-Lundqvist C, Mazzanti A, Blom N, Borggrefe M, et al. (2015) 2015 ESC guidelines for the management of patients with ventricular arrhythmias and the prevention of sudden cardiac death. Europace 17(11): 1601-1687.

56. Lane DA, Aguinaga L, Blomstöm-Lundqvist C, Boriani G, Dan GA, et al. (2015) Cardiac tachyarrhythmias and patient values and preferences for their management: the European Heart Rhythm Association (EHRA) consensus document endorsed by the Heart Rhythm Society (HRS), Asia Pacific Heart Rhythm Society (APHRS) and SOLEACE. Europace 17(12): 1747-1769.

57. Hoogwegt MT, Widdershoven JW, Theuns DA, Pedersen SS (2014) Information provision, satisfaction and emotional distress in patients with an implantable cardioverter-defibrillator. Int J Cardiol 177(2): 586-588.

Your next submission with Juniper Publishers
will reach you the below assets
- Quality Editorial service
- Swift Peer Review
- Reprints availability
- E-prints Service
- Manuscript Podcast for convenient understanding
- Global attainment for your research
- Manuscript accessibility in different formats
( Pdf, E-pub, Full Text, Audio)
- Unceasing customer service
Track the below URL for one-step submission
https://juniperpublishers.com/online-submission.php

